\title{
Formulations de Farines Composées Dont l'une à Base de Riz (Oryza Sativa) et L'autre à Base de Maïs (Zea Mays) Pour Enfants en âge de Sevrage
}

\author{
Loba Sonia Euphrasie, \\ Gbakayoro Jean Brice,
}

Unité de Formation et de Recherche des Sciences et Technologies des

Aliments, Laboratoirede Nutrition et Sécurité Alimentaire,

Université Nangui Abrogoua, Abidjan, Côte d'Ivoire

Kouame Kouassi Appolinaire,

Unité de Formation et de Recherche des Sciences Biologique, Laboratoire de

Biochimie, Université Péléforo Gon Coulibaly, Korhogo, Côte d'Ivoire

\section{Grodji Gbogouri Albarin, Brou Kouakou,}

Unité de Formation et de Recherche des Sciences et Technologies des

Aliments, Laboratoirede Nutrition et Sécurité Alimentaire,

Université Nangui Abrogoua, Abidjan, Côte d'Ivoire

Doi:10.19044/esj.2019.v15n33p100 URL:http://dx.doi.org/10.19044/esj.2019.v15n33p100

Résumé

La qualité des farines infantiles utilisées pendant la période de sevrage du nourrisson est d'une grande importance. L'objectif de cette étude est de mettre au point deux formulations de farine infantile dont l'une fabriquée à partir de riz enrichi en soia, en huile de palme et en sucre (FRSHS) et l'autre (FMHHS) à partir de maïs enrichi en haricot, en huile de palme et en sucre. Pour ce fait, les doses des ingrédients pour la formulation des deux farines (FRSHS et FMHHS) ont été combinées selon lesrecommandations nutritionnelles.Lescaractéristiques physico-chimiques, microbiologiques et organoleptiques de ces farines ont été déterminées selon les méthodes standards. Les résultats révèlent un taux de satisfaction de plus de $100 \%$ pour l'énergie et les protéines comparé aux normes nutritionnelles. Les teneurs respectives en lipides $(12,40 \pm 0,99 \%$ et $9,65 \pm 0,01 \%)$ pour les farines (FRSHS et FMHHS) sont comprises dans l'intervalle des valeurs recommandées et les taux de fibres des farines sont inférieurs à $5 \mathrm{~g}$, tel que recommandé. Les teneurs en fer $(11,19 \pm 0,12$ et $14,27 \pm 0,07 \mathrm{mg} / 100 \mathrm{~g}$ de matières sèche), de zinc $(4,91 \pm 0,05$ et $6,29 \pm 0,35 \mathrm{mg} / 100 \mathrm{~g}$ de matières sèche) et de calcium $(358,194 \pm 0,09$ et $343,87 \pm 0,44)$ des farines sont au-delà de la recommandation. Aussi, les farinesprésentent-elles de bonnes teneurs en la plupart des minéraux. Les charges microbiologiques détectées dans ces farines sont inférieures aux critères microbiologiques applicables aux farines infantiles. Les bouillies préparées à partir des farines composées ont été appréciées par les mères panelistes. Toutefois, la bouillie préparée à partir de la farine FRSHS a été plus acceptée. L'acceptabilité de la bouillie de FRSHS 
par rapport à celle de FMHHS par les mères pour leurs enfants se justifie par la couleur, l'arôme et le goût de la bouillie de farine FRSHS. Ces farines composées pourraient alors être recommandées pour les nourrissons, contribuant ainsi à lutter contre la malnutrition infantile.

Mots-Clefs : Farine infantile, Qualité nutritionnelle, Qualité microbiologique, Qualité organoleptique, Sevrage

\title{
Composite Flours Formulations, One Based on Rice (Oryza sativa) and the Other Based on Corn (Zea mays) for Children of Weaning Age
}

\author{
Loba Sonia Euphrasie, \\ Gbakayoro Jean Brice,
}

Unité de Formation et de Recherche des Sciences et Technologies des

Aliments, Laboratoirede Nutrition et Sécurité Alimentaire,

Université Nangui Abrogoua, Abidjan, Côte d'Ivoire

Kouame Kouassi Appolinaire,

Unité de Formation et de Recherche des Sciences Biologique, Laboratoire de

Biochimie, Université Péléforo Gon Coulibaly, Korhogo, Côte d'Ivoire

\section{Grodji Gbogouri Albarin, Brou Kouakou,}

Unité de Formation et de Recherche des Sciences et Technologies des

Aliments, Laboratoirede Nutrition et Sécurité Alimentaire,

Université Nangui Abrogoua, Abidjan, Côte d'Ivoire

\section{Abstract}

The quality of infant flours used during the infant weaning period is of great importance. The purpose of this study was to formulate infantile flours to cover the nutritional needs of children during weaning to fight against child malnutrition. Two types of flours were formulated, one made from rice enriched with soy, palm oil and sugar and the other made from maize enriched with beans, palm oil and sugar. Amounts and ingredients were combined as recommended. The physico-chemical, microbiological and organoleptic characteristics of these flours were determined according to standard methods. The results obtained showed a satisfaction rate of over $100 \%$ for energy and protein compared to nutritional standards. The lipid levels obtained (12.40 \pm 0.99 and $9.65 \pm 0.01$ ) respectively for the flours FRSHS and FMHHS were in the range of the recommended values and the fiber levels of the flours were less than $5 \mathrm{~g}$, as recommended. The iron contents $(11.19 \pm 0.12$ and $14.27 \pm$ 
$0.07 \mathrm{mg} / 100 \mathrm{~g}$ dry matter) and zinc $(4.91 \pm 0.05$ and $6.29 \pm 0.35 \mathrm{mg} / 100 \mathrm{~g}$ of dry matter) flours were largely above the recommendation. The microbiological loads detected in these flours were below the microbiological criteria applicable to infant flours. All the boils prepared from the compound flours were appreciated. The acceptability of the FRSHS boil compared to that of the FMHHS boil by mothers for their children is justified by the color, aroma and taste of the FRSHS boils most appreciated by the mothers. These formulations could then be recommended for infants, thus contributing to the fight against child malnutrition.

Keywords: Infant flour, Nutritional quality, Microbiological quality, Organoleptic quality, Weaning

\section{Introduction}

La malnutrition protéino-énergétique affecte approximativement 170 million d'enfants de moins de 5 ans, avec une prévalence de $40 \%$ en Asie du sud et 50\% en Afrique subsaharienne (De Oniset al.,2003). En Côte D'Ivoire près de $30 \%$ d'enfants souffrent de la malnutrition chronique, $8 \%$ sont émaciés et $15 \%$ ont une insuffisance pondérale (UNICEF, 2014). Toutes ces formes de malnutritions entraînent l'accroissement de la mortalité, l'élévation de la sensibilité aux maladies, la diminution des performances intellectuelles, la réduction des capacités physiques. En conséquent, le jeune enfant devenu adulte, est incapable de participer au développement socioéconomique du pays (OMS, 2000). Ces problèmes nutritionnels sont plus sévères en Afrique pendant la période de l'alimentation complémentaire (6 à 24 mois), au moment où le lait maternel n'arrive plus à couvrir les besoins nutritionnels de l'enfant en croissance (OMS/FAO, 2006). En effet, de la naissance à l'âge de 6 mois, tous les besoins nutritionnels de l'enfant sont couverts par le lait maternel. Audelà de cet âge, le lait maternel ne suffit plus pour couvrir entièrement les besoins en énergie et en protéines. C'est la période dite de sevrage qui s'étend de 6 mois à 1 an voire 2 ans. Pendant cette période, il faut apporter des aliments nouveaux sous forme liquide ou semi liquide pour compléter les apports du lait maternel. Ces nouveaux aliments donnés aux enfants pendant la période de sevrage sont appelés aliments de compléments et doivent être de qualité nutritive, sanitaire et organoleptique satisfaisante (Salle, 2009). La qualité des aliments de compléments utilisés pendant cette période est de ce fait d'une grande importance. En Afrique, pendant le sevrage, les mères nourrissent généralement leurs enfants avec des bouillies traditionnelles préparées à partir de farines simples ou composées provenant de céréales, de tubercules qui sont des aliments riches en glucides et pauvres en protéines. Ces aliments sont incapables de couvrir tous les besoins nutritionnels des enfants pendant le sevrage (Kouassiet al., 2015). Les besoins nutritionnels de l'enfant pendant 
le sevrage ont fait donc l'objet de nombreux travaux et de recommandations de l'OMS, conduisant à la fabrication de divers types de farines infantiles en vue de proposer aux enfants des produits de bonne qualité nutritive et sanitaire (Salle, 2009). En Afrique, ces farines infantiles de bonne qualité existent sur le marché mais ce sont des produits industriels importés et d'un coût élevé. Par conséquent, elles ne sont pas accessibles à toutes les bourses. La formulation d'aliments de complément à base de matière première locale répondant aux recommandations nutritionnelles et sanitaires s'avère une alternative efficace aux farines industrielles, fabriquées-en général à partir de produits importés qui coûtent chères(OMS/FAO, 2006). L'objectif de cette étude est de mettre au point deux formulations de farines à partir de matières premières locales dont l'une composée de riz, de soja et l'autre de maïs, de haricot, toutes deux enrichies en huile de palme et en sucre présentant une valeur nutritive, une qualité sanitaire adéquate, et acceptable par les enfants en âge de complémentation alimentaire.

\section{Matériel et méthodes \\ Matérielbiologique}

Le matériel végétal de l'étude est constitué des grains de riz (Oryzasativa), de soja (Glycine max), de maïs (Zeamays), de haricot (Phaseolusvulgaris), de l'huile de palme (Elaeis guineensis) et du sucre (Saccharum). Les grains de riz, de soja, de maïs, de haricot, l'huile de palme et le sucre ont été sélectionnées et achetées chez des grossistes au « Forum des marchés » d'Adjamé, commune d'Abidjan, capital économique de la Côte D’Ivoire. Le choix de ces céréales (riz, maïs) et légumineuses (haricot, soja) se justifie par le fait qu'ils sont disponibles en toute saison.

\section{Méthodes}

\section{Préparation des farines de riz, maïs, soja, haricot et formulation des farines (FRSHSet FMHHS)}

Les farines ont été produites selon le procédé standard (CAC, 2013) adapté aux caractéristiques des grains utilisées :

- triage, vannage des matières premières (riz, maïs, soja, haricot) premières;

- lavage des grains séparément;

- égouttage et séchage au soleil sur une surface propre en protégeant les matières premières avec du linge propre ;

- torréfaction des grains séparément à feu doux dans une poêle préalablement chauffé pendant dix minutes;

- mouture des grains séparément dans un moulin à disque préalablement nettoyé ;

- torréfaction des farines de riz, de maïs, de soja et de haricot ;

- refroidissement des différentes farines de riz, de maïs, de haricot et de soja ; 
- tamisage (tamis de 200 microns de diamètre) ;

- conditionnement dans des boîtes en plastique propre à l'abri de l'humidité

\section{Formulation des farines (FRSHS et FMHHS)}

La farine composée de riz et de soja enrichie en huile de palme et en sucre (FRSHS) et la farine composée de maïs et de haricot enrichie en huile de palme et en sucre (FMHHS) ont été élaborées à l'aide d'un système de formulation assisté par le logiciel Excel, en utilisant un système de calcul basé sur la méthode de formulation matricielle. A partir d'une liste d'ingrédients alimentaires, cette méthode permet de trouver la solution conduisant à couvrir au moins les besoins de deux nutriments avec au moins deux ingrédients (Olusayo et al.,2013 ; Afolayan, 2008). Le système d'équation utilisé est le suivant:

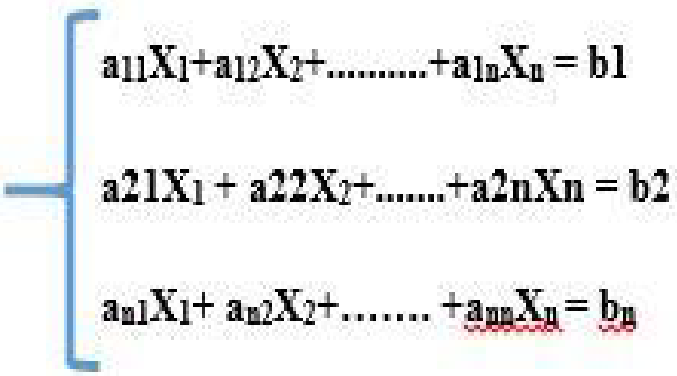

Avec $\mathrm{a}=$ les teneurs de nutriments (glucides, protéines, lipides et énergie) $\mathrm{X}=$ les proportions d'ingrédients à mélanger $\mathrm{b}=$ les besoins à satisfaire.

Ainsi les différentes valeurs de macronutriments (en g/100 g de farine : protéines, glucides, lipides) et d'énergie (en Kcal/100 g de farine) sont calculées automatiquement en fonction des formules de calculs élaborés sur le logiciel Excel. L'utilisation de cette méthode permet de formuler une farine infantile qui respecte les recommandations relatives à la teneur en nutriments (glucides, protéines et lipides) (CAC, 2013).Ainsi, 100 grammes de farine FRSHS ont été obtenus à partir de la combinaison de60 grammes de farine de riz, de 30 grammes de farine de soja, de 5 grammes d'huile de palme et de 5 grammes de sucre. Cent (100) grammes de farine (FMHHS) ont été obtenusà partir du mélange de 55 grammes de farines de maïs, de 35 grammes de farine de haricot, de 5 grammes d'huile de palme et de 5 grammes de sucre. Les farines développées (FRSHS et FMHHS) ont été standardisées en procédant à trois essais de la même formulation pour chaque formule de farine. Les farines obtenues ont été conditionnées dans les boîtes en plastique de manière à éviter les contaminations. 


\section{Analyse biochimique des farines composées FRSHS et FMHHS}

L'analyse physico-chimique des farines composées (FRSHS et FMHHS) a consisté en la détermination du taux d'humidité, de lipides, de protéines, de glucides, de cendre, de fibres brutes, de la valeur énergétique et de la composition minérale des farines. Les taux d'humidité, de lipides, de protéines, de glucides, de cendre, de fibres brutes et la valeur énergétique des farines composées ont été déterminés selon la méthode AOAC, (1990) en triple essai. Le taux d'humidité a été déterminé par séchage des échantillons dans une étuve (Memmert, Germany) à $105^{\circ} \mathrm{C}$ pendant 24 heures. La teneur en lipide a été déterminée en utilisant l'extraction par l'hexane dans un extracteur de type Soxhlet (UnidTecator, System HT2 1045, Suède). Après évaporation du solvant et séchage de la capsule à l'étuve (Memmert, Germany) à $105^{\circ} \mathrm{C}$ pendant $30 \mathrm{~min}$; la différence de poids a donné la teneur en lipides des échantillons.Le pourcentage de protéine brute a été déterminé en utilisant la méthode de Kjeldahl avec l'unité Kjeltec 8400 de l'analyseur (FOSS, Suède) et l'atome d'azote en pourcentage $(\% \mathrm{~N})$ obtenu est utilisé pour calculer le pourcentage de protéine brute $(\% \mathrm{P})$ en utilisant la relation suivante: $\% \mathrm{P}=\% \mathrm{~N} \times 6,25$

Le pourcentage de cendres (\%) a été déterminé en incinérant les échantillons de farines composées dans un four à moufle (NaberthermGmbH Babnhofstr.20,28865 Lilienthal/Bremen, Germany) à $600^{\circ} \mathrm{C}$ pendant 6 heures. La cendre est refroidie dans un dessiccateur et pesée. Pour les fibres brutes, $1 \mathrm{~g}$ d'échantillon de farine séchée est digéré avec de l'acide sulfurique 1,25 $\mathrm{N}$ et une solution d'hydroxyde de sodium 1,25 N. Le résidu insoluble obtenu est lavé à l'eau chaude et séché dans une étuve (Memmert 854 Schwabach, Allemagne) à $105^{\circ} \mathrm{C}$ pendant 24 heures. Le résidu séché est ensuite incinéré au four (NaberthermGmbHBabnhofstr. 20, 28865 Lilienthal/Bremen, Germany) à $600^{\circ} \mathrm{C}$ pendant 6 heures et pesé pour la détermination de la teneur en fibres brutes. La teneur en glucides (en \% de matière sèche) a été estimée par calcul différentiel à partir de la formule proposée par FAO (1998):

Taux de glucides $(\%$ MS $)=100-($ Protéines $(\%$ MS $)+$ Lipides $(\%$ $\mathrm{MS})+$ Cendre $(\% \mathrm{MS})+$ Fibres $(\% \mathrm{MS}))$

La valeur énergétique a été déterminée à partir du taux de lipides, de glucides et de protéines des farines composées en utilisant les facteurs de conversion d'Atwater; $4 \mathrm{Kcal} / \mathrm{g}$ pour les protéines, $9 \mathrm{Kcal} / \mathrm{g}$ pour les lipides et $4 \mathrm{Kcal} / \mathrm{g}$ pour les glucides (AOAC, 1990). La valeur énergétique en Kcal/100 $\mathrm{g}$ de matière sèche $(\mathrm{MS})$ a été calculéeà partir de la formule suivante:

Valeur Energétique $(\mathrm{Kcal} / 100 \mathrm{~g}$ de $\mathrm{MS})=(\%$ protéines $\times 4)+(\%$ glucides $\times 4)+(\%$ lipides 9$)$

La détermination des fractions minérales a été faite par dosage au spectrophotomètre d'absorption atomique des cendres des échantillons de 
farines composés; la lecture de la densité optique a été réalisée à $430 \mathrm{~nm}$ (AFNOR, 1977).

\section{Analyse microbiologique}

L'analyse microbiologique des farines (FRSHS et FMHHS) ont été basées sur la recherche des microorganismes susceptibles d'altérer la qualité hygiéniques et/ou nutritionnelle des farines. Il s'agit des germes tels que la flore aérobie mésophile, les coliformes thermotolérantset totaux, les levures et moisissures, Staphylococcus aureus et Salmonella. Les échantillons analysés ont été prélevées juste après obtention de chaque farine composée. Dix grammes de chaque farine ont été prélevés, auxquels $90 \mathrm{~g}$ d'eau peptonnéesalée (EPS) a été ajouté. Le mélange a été homogénéisé pendant 3 minutes pour obtenir la solution mère. Chaque dilution décimale est ensemencée en double sur des milieux de culture spécifiques pour la numération des germes retenus (NM 08.0.100, 2001). La flore aérobie mésophile totale, coliformes totaux etthermotolérants, levures et moisissures ont été dénombrés selon les procédures proposées par (Kumari et Ichhpujani, 2000). Les germes aérobies mésophiles totaux ont été dénombrés en milieu PCA (plate count agar) après 24 heures d'incubation à $37^{\circ} \mathrm{C}$. Les levures et moisissures ont été dénombrées sur gélose de Sabouraud en boîte de pétrie après incubation pendant 24 heures à $37^{\circ} \mathrm{C}$. Les coliformes totaux et fécaux ont été dénombrés en milieu VRBG (Violet Red Bile Gélose agar) après 24 heures d'incubation à $37{ }^{\circ} \mathrm{C}$ pour les coliformes totaux et $44{ }^{\circ} \mathrm{C}$ pour les coliformes fécaux pendant 48 heures. Le dénombrement des Staphylococcus a été faite selon la norme (NM 08.01.104, 2004). Les Staphylococcus aureus ont été dénombrés en milieu Mannitol chapmanagard (Sharlaud) après 48 heures d'incubation à $37^{\circ} \mathrm{C}$. Les salmonelles ont été dénombrées à partir d'une solution mère qui a été préparée à partir de $25 \mathrm{~g}$ de farines et $225 \mathrm{ml}$ d'eau peptonnée selon la norme (NM 08.0.116, 2004), qui a consisté à un préenrichissement en milieu non sélectif à $37^{\circ} \mathrm{C}$ pendant 24 heures et un enrichissement en milieu sélectif à $44^{\circ} \mathrm{C}$ pendant 24 heures.

\section{Evaluation de la qualité organoleptique des bouillies préparées à partir des farines composées (FRSHS et FMHHS)}

Des bouillies infantiles ont été préparées à partir de deux farines composées. Ainsi, dans une casserole, une quantité de $45 \mathrm{~g}$ de farine a été délayée dans $200 \mathrm{ml}$ d'eau puis cuite dans $350 \mathrm{ml}$ d'eau potable porté à ébullition. Le mélange a été maintenu au feu pendant 15 minutes sous agitation manuelle afin de laisser bien faire cuire le soja et réduire la teneur en facteurs antinutritionnels. Un panel non entraîné de 60 mères d'enfants de 6 à 24 mois a été recruté sur la base de leur disponibilité pour réaliser une série de tests sur les bouillies préparées. Les échantillons de bouillies codés ont été présentés 
simultanément à chaque panéliste dans un ordre randomisé. L’appréciation de chaque bouillie a été basée sur l'aspect (couleur), le goût (saveur), l'odeur (arôme), la consistance et l'acceptabilité globale des bouillies en utilisant une échelle hédonique à neuf (9) points (AFNOR, 2000). Le chiffre 9 correspond à un produit extrêmement agréable, le 8 est attribué à un produit très agréable. Le chiffre 7 correspond à un produit agréable, le 6 à un produit assez agréable. Le chiffre 5 est attribué à un produit ni agréable ni désagréable (ni bon ni mauvais), le 4 correspond à un produit assez désagréable. Le chiffre 3 correspond à un produit désagréable, le 2 est attribué à un produit très désagréable et le 1 à un produit extrêmement désagréable.

\section{Analyses statistiques}

L’analyse de variance (ANOVA) a été effectuée avec le logiciel Statistica version 7.1 pour étudier le degré de différence entre les variables. En cas de différence significative entre les paramètres étudiés, le classement des moyennes (groupes homogènes) est effectué avec le test de Duncan. Le seuil de signification $(\alpha)$ est de 0,05 . Les différences statistiques avec une valeur de probabilité inférieure à 0,05 ont été considérées comme significatives.

\section{Résultats}

Composition en macronutriments, teneur en cendres et valeur énergétique des farines infantiles (FRSHS et FMHHS)

Les teneurs des principaux macronutriments, des cendres et les valeurs énergétiques des farines infantiles (FRSHS et FMHHS) sont récapitulées dans leTableau 1. Les taux d'humidité, des macronutriments, de cendres et les valeurs énergétiques des farines (FRSHS et FMHHS) sur la base de la matière sèche $(\mathrm{MS})$ diffèrent significativement entre elles $(\mathrm{P}<0,05)$. A l'exception donc de la teneur en glucide, la farine FRSHS a présenté les taux de nutriments les plus élevés par rapport à la farine FMHHS. La farine FRSHS contient $12,405 \%$ de lipides ; 20,435 \% de protéines ; 61,01 \% de glucides pour une valeur énergétique qui est de $437,425 \mathrm{kcals}$ pour $100 \mathrm{~g}$ de matière sèche tandis que la farine FMHHS contient 9,65\% de lipides ; 16,78\% de protéines ; 67,87 $\%$ de glucides pour une valeur énergétique qui est de 425,45 kcals pour $100 \mathrm{~g}$ de matière sèche. La teneur en fibre est de 3,42 g/100 MS dans la farine (FRSHS) contre 3,28 g/100 MS dans la farine (FMHHS). Le taux d'humidité est plus élevé dans la farine (FRSHS) par rapport à la farine (FMHHS) (P $<0,05)$. Les taux d'humidité sont respectivement de 4,78 g/100 MS et 4,38 g/100 MS. La teneur en cendre la plus élevée est obtenue avec la farine FRSHS $(2,73 \mathrm{~g} / 100 \mathrm{MS})$ tandis que le plus faible taux est obtenu avec la farine (FMHHS) $(2,42$ g/100 MS). 
Tableau 1 : Teneurs en macronutriments, en cendres et valeur énergétique des farines

(FRSHS et FMHHS)

\begin{tabular}{|c|c|c|}
\hline \multirow{2}{*}{ Paramètres } & \multicolumn{2}{|c|}{ Farines } \\
\cline { 2 - 3 } & FRSHS & FMHHS \\
\hline Humidité (g/100 MS) & $4,78 \pm 0,001^{\mathrm{a}}$ & $4,38 \pm 0,025^{\mathrm{b}}$ \\
\hline Lipides (g/100 MS) & $12,405 \pm 0,995^{\mathrm{l}}$ & $9,65 \pm 0,015^{\mathrm{k}}$ \\
\hline Protéines (g/100 MS) & $20,435 \pm 0,305^{\mathrm{m}}$ & $16,78 \pm 0,16^{\mathrm{n}}$ \\
\hline Cendre (g/100 MS) & $2,73 \pm 0,02^{\mathrm{k}}$ & $2,42 \pm 0,001^{\mathrm{m}}$ \\
\hline Fibres (g/100 MS) & $3,42 \pm 0,01^{\mathrm{a}}$ & $3,28 \pm 0,001^{\mathrm{k}}$ \\
\hline Glucides (g/100 MS) & $61,01 \pm 0,06^{\mathrm{k}}$ & $67,87 \pm 0,03^{\mathrm{m}}$ \\
\hline $\begin{array}{c}\text { Valeur énergétique } \\
\text { (Kcal/100 g de MS) }\end{array}$ & $437,42 \pm 0,034^{\mathrm{g}}$ & $425,45 \pm 0,03^{\mathrm{b}}$ \\
\hline
\end{tabular}

Toutes ces valeurs sont les moyennes de trois déterminations.Les valeurs portant les lettresdifférentes dans une ligne sont significativement différentes au seuil de $5 \%$.

\section{Composition en minéraux des farines (FRSHS et FMHHS)}

La composition minérale des farines est présentée dans le Tableau 2. Les teneurs en minéraux de la farine FRSHS diffèrent statistiquement de celles de la farine de FMHHS $(\mathrm{P}<0,05)$. Ainsi, les taux de magnésium, de phosphore, de calcium, de potassium et de cuivre ont été plus élevés dans la farine FRSHS que dans la farine de FMHHS. Les taux sont respectivement de 95,$82 ; 286,48 ; 358,194 ; 440,57 ; 6,006 \mathrm{mg} / 100 \mathrm{~g}$ de matière sèche pour la farine FRSHS contre 84,94; 192,39; 343,87; 356,56; 1,694 mg/100g de matière sèche pour la farine FMHHS. Les taux de sodium, de fer et de zinc ont été plus élevés dans la farine FMHHS que dans la farine FRSHS. Les taux ont été respectivement de 25,08; 14,27 et de 6,292 mg/100g de matière sèche pour la farine de FMHHS contre 20,09; 11,19; 4,91 mg/100g de matière sèche pour la farine FRSHS. 
Tableau 2 : Composition minérale des farines FRSHS et FMHHS

\begin{tabular}{|l|c|c|}
\hline \multirow{2}{*}{ Paramètres } & \multicolumn{2}{|c|}{ Farines } \\
\cline { 2 - 3 } & FRSHS & FMHHS \\
\hline Sodium (Na) (mg/100 MS) & $20,09 \pm 0,03^{\mathrm{a}}$ & $25,08 \pm 0,09^{\mathrm{b}}$ \\
\hline Magnésium (Mg) (mg/100 MS) & $95,82 \pm 0,23^{1}$ & $84,94 \pm 0,29^{\mathrm{k}}$ \\
\hline Phosphore (P) (mg/100 MS) & $286,48 \pm 0,44^{\mathrm{m}}$ & $192,39 \pm 0,30^{\mathrm{a}}$ \\
\hline Potassium (K) (mg/100 MS) & $440,57 \pm 0,37^{\mathrm{h}}$ & $356,56 \pm 0,94^{\mathrm{J}}$ \\
\hline Calcium (Ca) (mg/100 MS) & $358,194 \pm 0,09^{\mathrm{o}}$ & $343,87 \pm 0,44^{\mathrm{P}}$ \\
\hline Fer (Fe) (mg/100 MS) & $11,19 \pm 0,12^{\mathrm{d}}$ & $14,27 \pm 0,07^{\mathrm{e}}$ \\
\hline Cuivre (Cu) (mg/100 MS) & $6,006 \pm 0,09^{\mathrm{g}}$ & $1,694 \pm 0,12^{\mathrm{x}}$ \\
\hline Zinc (Zn) (mg/100 MS) & $4,91 \pm 0,05^{\mathrm{f}}$ & $6,292 \pm 0,35^{\mathrm{s}}$ \\
\hline
\end{tabular}

Toutes ces valeurs sont les moyennes de trois déterminations. Les valeurs portant les lettres différentes dans une ligne sont significativement différentes au seuil de $5 \%$.

\section{Qualité microbiologique des farines FRSHS et FMHHS}

Le Tableau 3 présente la qualité microbiologique des farines FRSHS et FMHHS. La charge fongique des farines varie de 2,1.101 à 5,6.101 UFC/g. La charge des germes aérobies mésophiles varie de 2,2.102 à 9,6.102 UFC/g. La charge des coliformes totaux varie de 10 à $12 \mathrm{UFC} / \mathrm{g}$. Les coliformes thermotolérants, Staphylococcus aureus et les salmonelles n'ont pas été détectés dans les farines. Les charges fongiques et bactériennes détectées dans les farines sont inférieures aux critères microbiologiques applicables aux farines infantiles (Codex Alimentarius, 2013).

Tableau 3: Qualité microbiologique des farines FRSHS et FMHHS

\begin{tabular}{|l|l|l|l|l|l|l|}
\hline \multirow{2}{*}{ Farines } & \multicolumn{6}{l}{ Germes (UFC/grammed'aliments) } \\
\cline { 2 - 7 } & GAM & CT & CF & Staph & Sal & Lev et Mois \\
\hline FRSHS & $2,210^{2} \pm 2,11^{\mathrm{f}}$ & $12 \pm 1,01^{\mathrm{o}}$ & $\begin{array}{l}0,0 \\
0,0^{\mathrm{t}}\end{array}$ & $\pm, 0 \pm 0,0^{\mathrm{k}}$ & Absent & $2,1.10^{1} \pm 0,07^{\mathrm{h}}$ \\
& & & $\begin{array}{l}0,0 \\
0,0^{\mathrm{t}}\end{array}$ & $0,0 \pm 0,0^{\mathrm{k}}$ & Absent & $5,6.10^{1} \pm 0,11^{\mathrm{p}}$ \\
\hline FMHHS & $9,610^{2} \pm 1,8^{\mathrm{e}}$ & $10 \pm 1,01^{\mathrm{p}}$ & & & \\
\hline Norme & $<10^{5}$ & $<10^{2}$ & $<10^{2}$ & $<10^{2}$ & Absent & $<10^{3}$ \\
\hline
\end{tabular}


Toutes ces valeurs sont les moyennes de trois déterminations. Les valeurs portant les lettres différentes dans une même colonne sont significativement différentes au seuil de 5\%. GAM : Germes aérobies mésophiles, CT: Coliformes totaux, CF : Coliformes fécaux, Staph : Staphylococcus aureus, Sal: les salmonelles, Lev et Mois: Levure et moisissures.

\section{Caractéristique organoleptique des bouillies des farines FRSHS et FMHHS}

Le Tableau 4 présente les résultats des tests organoleptiques sur les bouillies de farines FRSHS et FMHHS. Au niveau du goût, l'arôme, la couleur et l'acceptabilité globale, la préférence des mères est significative différente $(P<0,05)$ entre les bouillies, tandis que pour le paramètre consistance il n'y a pas de différence significative $(\mathrm{P}>0,05)$. Pour les paramètres (goût, arôme, couleur et acceptabilité globale), la bouillie de farine FRSHS a obtenu des moyennes allant de 6,41 à 7,66. Ces notes correspondent, en utilisant l'échelle hédonique, à un produit apprécié dans l'intervalle assez agréable à agréable. La bouillie de farine FMHHS a obtenu des moyennes allant de 5,33 à 6,33 pour les paramètres (goût, arôme, couleur et acceptabilité global). Ces notes correspondent, en utilisant l'échelle hédonique, à un produit apprécié dans l'intervalle ni agréable ni désagréable à un produit assez agréable.

Tableau 4 : Caractéristiques organoleptiques des bouillies de FRSHS et FMHHS

\begin{tabular}{|c|c|c|c|c|c|}
\hline Bouillies & Goût & Couleur & Arôme & Consistance & $\begin{array}{l}\text { Acceptabilité } \\
\text { global }\end{array}$ \\
\hline FRSHS & $6,95 \pm 0,28^{\mathrm{k}}$ & $6,41 \pm 0,19^{\mathrm{a}}$ & $7,66 \pm 0,21^{\mathrm{m}}$ & $5,14 \pm 0,26^{\mathrm{c}}$ & $7,33 \pm 0,18^{\mathrm{J}}$ \\
\hline FMHHS & $5,13 \pm 0,33^{\mathrm{m}}$ & $5,33 \pm 0,42^{\mathrm{b}}$ & $5,33 \pm 0,21^{\mathrm{P}}$ & $5,18 \pm 0,34^{\mathrm{c}}$ & $6,33 \pm 0,14^{\mathrm{l}}$ \\
\hline
\end{tabular}

Les valeurs portant les lettres différentes sur une colonne sont significativement différentes au seuil de $5 \%$.

\section{Discussion}

La présente étude a pour objectif de proposer à des ménages généralement à faibles revenus, des farines infantiles de bonne qualité provenant de produits locaux disponibles et accessibles. De ce fait deux formules de farines ont été élaborées dont l'une à base de riz enrichit en soja, en huile de palme et en sucre (FRSHS) et l'autre à base de maïs enrichit en haricot, en huile de palme et en sucre. L'incorporation des protéines végétales, particulièrement le soja et le haricot dans la formulation des farines se justifient par le fait qu'elles sont peu coûteuses et disponibles par rapport aux autres protéines animales (OMS/UNICEF, 2003). L'addition du sucre et de l'huile dans les différentes farines a été effectuée pour modifier sensiblement la viscosité des bouillies issues des formulations de farines et améliorer le goût 
des bouillies (Trèche, 1998). Aussi, ces farines composées en combinant au moins quatre groupes d'aliments (céréales, légumineuses, produit sucré et matières grasses) sur les sept recommandés peuvent contribuer en termes de qualité à la diversité alimentaire minimale préconisée pour les enfants de 6 à 24 mois (WHO, 2008). La torréfaction a été effectuée pour la formulation des farines comme technique de précuisons pour réduire les facteurs antinutritionnels, inactiver les facteurs antitrypsine du soja et réduire la flore microbienne des farines (Saskia et Annoek, 2005).

Le taux d'humidité compris entre 4,38 et 4,78\% des farines FRSHS et FMHHS est satisfaisante, la norme indiquant un taux de 5\% dans les farines infantiles (FAO/OMS, 2006). Ces résultats sont en accord avec ceux issu des travaux de Aloziéet al., (2009) qui ont signalé dans la farine infantile de bambara un taux d'humidité de 5\%, valeur au-delà de laquelle le milieu devient favorable au développement des microorganismes. Les farines enrichies de cette étude peuvent ainsi être bien conservées. Les teneurs en glucides des farines enrichies obtenues (61,01 et 67,87\%) respectivement pour les farines FRSHS et FMHHS sont en accord avec la norme estimée à 68\% (FAO/OMS, 2006). Aussi, les teneurs en protéines renseignées dans cette étude $(20,43$ et $16,78 \%)$ sont conformes à la norme estimée à environ(15\%) (FAO/OMS, 2006). Les teneurs en protéines des farines formulées sont avantageuses pour la croissance de l'enfant car les protéines auraient un impact positif sur la réparation des tissus et la musculation (Soroet al., 2013). L'apport lipidique dans les farines infantiles destinées aux enfants pendant le sevrage doivent représenter environ $30 \%$ de l'apport total énergétique pour un taux d'environ $8 \%$ (Lutter et $a l ., 2003$; FAO/OMS, 2006). Les farines composées FRSHS et FMHHS ont produit respectivement des taux de 12,40 et $9,65 \%$ de lipides, représentant respectivement 23 et $21 \%$ de l'apport énergétique total des farines. De ce fait, l'apport en lipide des farines composées FRSHS et FMHHS est satisfaisant. Les teneurs en fibres des farines infantiles ne doivent pas excéder $5 \mathrm{~g}$ pour $100 \mathrm{~g}$ de produit sur la base de la matière sèche (FAO/OMS, 2006). Les valeurs obtenues dans ces farines composées sont donc conformes à la norme avec des taux de $(3,42$ et 3,28\%). Cette faible teneur en fibre des farines pourrait réduire l'encombrement de la nourriture et favoriser une haute digestibilité et l'absorption des nutriments tels que les protéines et les minéraux comme signalé par Olorufémiet al., (2006). Conformément aux travaux de AFSSA, (2002) la présence des fibres dans ces farines composées est favorable à la diminution de la glycémie et de l'insulinémie post-prandiale. L'énergie est l'un des éléments les plus déterminants dans la croissance des nourrissons. Les valeurs moyennes d'énergie de 437 et $425 \mathrm{Kcal}$ pour $100 \mathrm{~g}$ de matière sèche renseignées dans les farines enrichies de cette étude sont inférieure à celles rapportées par les travaux de Franck, (2015) dans les farines infantiles de ceréale-legumineuse 
préparées par voie humide et sèche, mais sensiblement proche à la norme qui est de $400 \mathrm{Kcal}$ (Mouquetet al., 2008).Concernant, la composition minérale des farines FRSHS et FMHHS, les taux de magnésium, de potassium, de fer, de cuivre et de zinc sont supérieure aux valeurs minimum recommandées (FAO/OMS, 2006). Les valeurs minimum de conformité étant respectivement de 48,7; 408,7; 8,5; 0,1 et 3,7 mg pour $100 \mathrm{~g}$ de matières sèche. Aussi les deux farines enrichies ont un taux de calcium conforme à la valeur recommandée de 341,2 mg/100g de matière sèche (FAO/OMS, 2006). Comparativement au taux de phosphore recommandé pour les farines infantiles qui est de 281,2mg/100g de matière sèche (CAC, 2013), seul la farine de FRSHS contient un taux satisfaisant de $286,48 \mathrm{mg} / 100 \mathrm{~g}$ de matière sèche.Toutefois la présence de tous ces minéraux dans ces farines formulées serait bénéfique car ils interviennent dans le développement de l'organisme de l'enfant et peuvent limiter l'apparition de carences nutritionnelles comme signalé avec les travaux de Lutter et al., (2003). En effet, le fer intervient dans la constitution de l'hémoglobine, de la myoglobine et de nombreuses enzymes, il est aussi indispensable pour un grand nombre de réactions métaboliques. Le calcium et le phosphore assurent quant à eux la rigidité des os et favorisent la croissance des enfants pendant le sevrage. Le zinc représente avec le fer les minéraux les plus concentrés au niveau du cerveau. Aussi, il est indispensable de couvrir les besoins en zinc chez le nourrisson où la croissance cérébrale est encore importante. Le zinc est aussi impliqué dans l'immunité car il réduit l'incidence et la gravité des diarrhées chez l'enfant. Le cuivre en très faible quantité dans le corps humain entre dans la composition de nombreux enzymes et intervient également dans le phénomène de minéralisation osseuse, de renforcement de l'immunité et dans les métabolismes du fer et du glucose (Lokombéet al., 2004). Le magnésium quant à lui est nécessaire pour les réactions biochimiques dans le corps, permettant de maintenir le muscle, améliorant le fonctionnement du nerf, le maintien du rythme cardiaque et la régulation du taux de sucre dans le sang (Lutter et al., 2003). La charge des germes aérobies mésophiles et des champignons varie respectivement de $2,2.10^{2}$ à $9,6 \cdot 10^{2} \mathrm{UFC} / g$ et de $2,1 \cdot 10^{1}$ à 5,6.10 ${ }^{1} \mathrm{UFC} / \mathrm{g}$. Ces différentes charges sont inférieures aux critères microbiologiques concernant les germes aérobies mésophiles et les champignons respectivement de $10^{5} \mathrm{UFC} / \mathrm{g}$ et de $10^{3} \mathrm{UFC} / \mathrm{g}$ dans les farines infantiles (CAC, 2013). Ces faibles charges pourraient s'expliquer par le taux d'humidité très bas dans les différentes farines composées FRSHS et FMHHS. En effet, ce taux d'humidité obtenu serait dû au séchage préalable des grains et des farines de riz, maïs, haricot et soja. Aussi, le couplage torréfaction/séchage aurait permis une réduction significative de la charge des germes aérobies mésophiles et des champignons dans les farines de cette étude conformément aux travaux de Tarhouniet al., (2015). Néanmoins la présence des moisissures dans ces farines composées 
pourrait être la cause d'intoxication alimentaire au cas où une moisissure toxinogène secréterait une toxine insensible à la chaleur comme démontré par certains auteurs tels que Tabauc, (2007). Les coliformes totaux, les coliformes thermotolérants, les staphylocoques et les salmonelles n’ont pas été détectés dans les farines composées. Ces résultats microbiologiques obtenus traduisent le respect des bonnes pratiques d'hygiène au cours de la fabrication des farines. Les différentes farines obtenues dans cette étude sont donc de qualité microbiologique satisfaisante. En dehors de l'équilibre nutritionnel et de l'innocuité des farines infantiles, leur qualité sensorielle a été également appréciée, étant une condition essentielle de l'acceptabilité des aliments par les consommateurs (Corinne, 2013). La bouillie obtenue à partir de la formule de farine FRSHS a été plus acceptée par les mères sur la base de sa couleur, de son arôme et de son goût que la bouillie formulée à partir de la farine FMHHS.

\section{Conclusion}

Cette étude a permis de mettre au point deux farines infantiles enrichies en ressources alimentaires locales répondant aux recommandations de l'OMS en termes de composition proximale, microbiologique et organoleptique pour l'alimentation complémentaire. En effet les farines contribuent en termes de qualité à la diversité alimentaire minimale préconisée pour les enfants de 6 à 24 mois, en combinant au moins quatre groupes d'aliments sur les sept recommandés. Ces deux farines composées (FRSHS et FMHHS) renferment de bonnes teneurs en protéines, en lipides, en glucides et en la plupartdes minéraux. En perspective, il est envisagé une évaluation de l'efficacité de ces farines enrichies sur les animaux afin d'apprécier la qualité des farines in vivo ainsi que leurs effets secondaires.

\section{Contributions des auteurs}

Sonia Euphrasie Loba a conçu le sujet, réalisé les analyses physicochimiques et organoleptiques, collecté les données et rédigé le manuscrit. AlbarinGrodjiGbogouri a réalisé l'échantillonnage, la fabrication des farines composées. Jean Brice Gbakayoro a réalisé les analyses statistiques. KouaméAppolinaireKouassi a corrigé le manuscrit. Kouakou Brou amonitoré le groupe pour toute la recherche.

\section{Remerciements}

Nous remercions le laboratoire de Nutrition et Sécurité alimentaire de l'Université Nangui Abrogoua pour avoir facilité les travaux de laboratoire et les analyses. Les mères de l'hôpital du district communautaire d'Abobo situé dans la ville d'Abidjan qui ont servi de paneliste. 


\section{References:}

1. De Onis M, Frongillo EA, Blössner M. (2000).Is malnutrition declining? An analysis of changes in levels of child malnutrition since 1980.Bull World Health Organ, 78 (10): 1222-33.

2. Fond des nations unies pour l'enfance. (2014). Analyse de la situation de l'enfant en Côte d'Ivoire, Draft ; pp 1-127.

3. Organisation Mondial de la Santé. (2000). Nutrition du nourrisson et du jeune enfant. Rapport du Directeur général: cinquante-troisième assemblée mondiale de la sante A53/7. Désormais accessible sur le site Web de l'OMS: http://www.who.int/nutgrowthdb.

4. FAO/OMS. (2006). Programme mixte FAO/OMS sur les normes alimentaires. Rapport des vingt -septième sessions du comité du codex sur la nutrition et les aliments diététiques ou de régime, ALINOM 06/29/26, pp 1-105.

5. SALLE B. (2009). Alimentation du nouveau-né et du nourrisson. Bulletin Académie Nationale de Médecine, 193 (2) : 431-446.

6. Kouassi KA, Adouko AE, Gnahe DA, Grodji GA, Kouakou B, Gnakri D. (2015). Comparaison des caractéristiques nutritionnelles et rhéologiques des bouillies infantiles préparées par les techniques de germination et de fermentation. International Journal of Biological and Chemical Sciences, Biological and Chemical Sciences, 9(2): 944-953. Desormais accessible sur : http://dx.doi.org/10.4314/ijbcs.v9i2.31.

7. CAC (Commission du Codex Alimentarius). (2013). Lignes directrices pour la mise au point des préparations alimentaires complémentaires destinées aux nourrissons du deuxième âge et aux enfants en bas âge, pp1-223.

8. Olusayo OE, Olusesan AB, Adesola AG. (2013). Review of Livestock Feed Formulation Techniques.Journal of Biology, Agriculture and Healthcare, 3(4): 60-77.

9. Afolayan MO, Afolayan M. (2008). Nigeria Oriented Poultry Feed Formulation Software Requirements. Journal of Applied Sciences Research, 4(11): 1596-1602.

10. AOAC (Association of Official Analytical Chemists). (1990). Official Methods of Analysis of the Association of Analytical Chemists (15th Edition), AOAC.Washington, DC, USA, 684pp.

11. FAO. (1998). Les aliments vendus sur la voie publique. Rome : 96p.

12. AFNOR. (1977). V18-101. Ressource électronique. Disponible sur http: www.afnor.org.

13. NF V08-0-100. (2001). Préparation des échantillons, de la suspension mère et des dilutions décimales en vue de l'examen microbiologique, pp14-28.

14. NM 08.0.116, 2004. Recherche des Salmonella, pp101-105. 
15. Kumari S, Ichhpujani RL. (2000). Guidelines on Standard Operating Procedures for Microbiology.WHO : Geneva, pp 304-316.

16. NM 08.01.104, ISO 6888. (2004). Directives générales pour le dénombrement de Staphylococcus aureus, pp 101-114.

17. NM 08.0.116. (2004). Recherche des Salmonella, pp 121-134.

18. AFNOR. (2000). Norme XP V09-500 en cours de révision. Directives générales pour la réalisation d'épreuves hédoniques en laboratoire d'évaluation sensorielle ou en salle en conditions contrôlées impliquant des consommateurs. In AFNOR, recueil de normes, Analyse sensorielle, 6ème édition, pp120- 168.

19. OMS/UNICEF. (2003). Alimentation complémentaire des jeunes enfants dans les pays en développement. OMS: Genève; pp 130-131.

20. WHO. (2008). Indicators for Assessing Infant and Young Child Feeding Practices. Conclusions of a Consensus Meeting held 6-8 November 2007 in Washington, DC, USA Geneva. Available at http://whqlibdoc.who.int/publications/2008/9789241596664_eng.pdf.

21. Saskia VG, Annoek VW. (2005). Production Artisanale d'aliments de complément, série AgrodokCordaid, N²2, Pays- Bas, pp224-276.

22. Alozie YE, Iyam MA, lawalo O, udofia U, Ani IF. (2009). Utilisation de la farine de Bambara comme aliment de complément. Journal of Food Technology, 7(4): 111-114.

23. Soro S, Konan G, Elleingand E, N'guessan D and E Koffi. (2013). Formulation d'aliments infantiles à base de farines d'igname enrichies au soja. African Journal of Food agriculture nutrition and development.3(5):8313 - 8339.

24. Lutter CK, Dewey KG.(2003). Proposed nutrient composition for fortified complementary foods.Journal Nutr,133(9): 3011S-20S.

25. Olorunfemi OB, Akinyosoye FA, Adetuyi FC. (2006). Microbial and nutritional evaluation of infant weaning food from mixture of fermented food substrates.Research Journal of Biological Science, 20 (1): 20-23.

26. AFASS. (2002). Les fibres alimentaires : définition méthodes de dosage allégations nutritionnelles. Rapport du comité des experts spéciales Nutrition humaine, 62pp.

27. Mouquet C, Icard VC, Guyot JP, Tou E, Rochette I, Trèche S. (2008). Consumption pattern, biochemical composition and nutritional value of fermented pearl millet gruels in Burkina Faso.International Journal of Food Sciences and Nutrition, 59 (12): 716-729.

28. Lokombé LA, Mullié C. (2004). Nutrition du nourrisson et diversification alimentaire. Cahiers de Nutrition et Diététique, 39 (12): 349-359. 
29. Tarhouni A, Djendoubi N, Amri F, Elbour M, Sadok S, Mihoubi BN. (2015). Mise au point d'un procédé intégré de valorisation de la sardinelle: effet de la température et du blanchiment sur la valeur nutritionnelle et de la qualité microbiologique des produits finis. Bulletin de l'Institut National des Sciences et Technologies de la Mer Salammbô, 42 (11): 69-71.

30. Tabauc C. (2007). Flore fongique de différentes substrats et condictions optimales de production de mycotoxines. Thèse de doctorat. Institut National Polytechnique de Toulouse et Université de Bucarest. P.86.

31. Corinne G. (2013). Additifs Alimentaires, Le Guide Indispensable pour ne plus vous Empoisonner. Ed. Chariot d'Or: Paris, France, pp 134- 166. 